\section{The J-ROCKET AF Study: A Matter of Ethnicity or a Matter of Weight? - Reply -}

We appreciate Dr Delgado-Fernández's comments on the JROCKET AF study. Clinical pharmacology studies have reported an approximately $30 \%$ higher rivaroxaban exposure in Japanese patients compared with Caucasian patients, which can be partly attributed to body weight differences; however, the difference in exposure cannot be totally explained in terms of body weight or body size. ${ }^{1,2}$ Moreover, no specific ethnic factor that could possibly be responsible for the exposure difference has been identified.

Based on the results of clinical studies, including clinical pharmacology studies conducted in other countries, renal function, body weight, and age have been identified as the factors associated with rivaroxaban exposure. However, given that age and body weight do not significantly affect rivaroxaban exposure, dose adjustment based on these factors is thought to be unnecessary. On the other hand, renal function is considered an essential factor for dose adjustment, considering the effect on exposure and the wide range of distribution in the target patient population. ${ }^{3}$ Additionally, assessment of renal function with creatinine clearance using the Cockcroft-Gault formula already takes, in addition to serum creatinine, age and body weight into consideration.

The dosage in the J-ROCKET AF study was established in consideration of the differences in medical practice between Japan and other countries. The Japanese guideline recommended that elderly Japanese have a lower INR requirement, in addition to the fact that exposure is higher in Japanese patients than in Caucasian patients. ${ }^{4}$ In the J-ROCKET AF study, the incidence of the primary safety endpoint in the population with $\mathrm{BMI} \leq 25$ and that in the population with BMI $>25$ was not significantly different in the rivaroxaban group, and the hazard ratios for rivaroxaban vs. warfarin were also comparable between the 2 populations (unpubl. data).

Regarding efficacy, there was also no difference in the size of the effect of rivaroxaban between subgroups stratified by BMI; however, given that the J-ROCKET AF study did not have adequate statistical power to validate efficacy, interpretation of subgroup analysis requires careful attention.

\section{References}

1. Kaneko M, Tanigawa T, Hashizume K, Kajikawa M, Tajiri M, Mueck W. Confirmation of model-based dose selection for Japanese phase III study of rivaroxaban in non-valvular atrial fibrillation patients. Drug Metab Pharmacokinet 2013 January 22, doi:10.2133/ dmpk.DMPK-12-RG-109 [Epub ahead of print].

2. Tanigawa T, Kaneko M, Hashizume K, Kajikawa M, Ueda H, Tajiri M, et al. Model-based dose selection for phase III rivaroxaban study in Japanese patients with non-valvular atrial fibrillation. Drug Metab Pharmacokinet 2013; 28: 59-70.

3. Mueck W, Lensing AW, Agnelli G, Decousus H, Prandoni P, Misselwitz F. Rivaroxaban: Population pharmacokinetic analyses in patients treated for acute deep-vein thrombosis and exposure simulations in patients with atrial fibrillation treated for stroke prevention. Clin Pharmacokinet 2011; 50: 675-686.

4. Group JCSJW. Guidelines for pharmacotherapy of atrial fibrillation (JCS 2008): Digest version. Circ J 2010; 74: 2479-2500.

Masatsugu Hori, MD, PhD

Osaka Medical Center for Cancer and Cardiovascular Diseases, Osaka, Japan

Mariko Kajikawa, MD, PhD

Bayer Yakuhin Ltd, Osaka, Japan

(Released online August 1, 2013) 